\title{
Harnessing autophagy for adoptive T-cell therapy
}

\section{“...through use of ex vivo methods of T-cell expansion that incorporate rapamycin and polarizing cytokines, autophagy can be harnessed for the generation of Th1- or Th2-type T cells that mediate increased in vivo effects upon adoptive transfer."}

\section{KEYWORDS: autophagy $\approx$ immunology $\approx$ rapamycin $\approx$ Th1 $=$ Th2 $\approx$ transplantation - tumor}

Allogeneic hematopoietic cell transplantation (HCT), which is a treatment option for leukemia and lymphoma (reviewed in [1]), represents a form of adoptive T-cell therapy (the graftversus-tumor effect). The majority of allogeneic transplants are performed using unmanipulated donor $\mathrm{T}$ cells, although as we will describe below, ex vivo-manipulated donor T-cell therapy represents a promising new area of clinical research. In addition, in the autologous setting, ex vivo-expanded $\mathrm{T}$ cells, in particular $T$ cells that are gene-modified to enhance tumor targeting or avoid viral infection, have been evaluated in a diversity of diseases including melanoma and sarcoma [2], leukemia [3] and HIV [4,5]. Successful adoptive T-cell therapy in part requires that the transferred $\mathrm{T}$ cells: differentiate into a favorable T-cell subset, which may minimally include Th1, Th2, Treg or Th17 populations depending on the clinical context [6]; and persist in vivo [7]. In this article, we will focus on the ex vivo use of rapamycin to generate functionally defined T-cell subsets that possess a favorable pattern of in vivo persistence after adoptive transfer. Current results indicate that rapamycin induces autophagy during ex vivo T-cell differentiation, with resultant T-cell acquisition of an antiapoptotic phenotype that confers in vivo persistence.

Autophagy is triggered during cellular stresses such as starvation, growth factor withdrawal or through rapamycin pharmacologic blockade of mTOR [8], which is a central gatekeeper of cell signaling pathways and cellular energetics. Three types of autophagy exist:

- Macroautophagy, which involves the enclosure of cytoplasm, cytoplasmic protein aggregates or whole organelles in a double membranebound structure that is degraded by lysosomes;

- Microautophagy, which is the capture of cytoplasmic contents in small vesicles that bud directly into lysosomes;

- Chaperone-mediated autophagy, which involves translocation of proteins containing a target sequence into the lysosomal membrane.

Rapamycin primarily results in macroautophagy (hereafter referred to as autophagy) that occurs in three stages:

- Initiation, which involves the formation of an isolation membrane around the cytoplasm;

- Maturation, where membrane expansion results in the formation of an autophagosomal double membrane;

- Degradation in the lysosomes.

These three events are successfully orchestrated by various molecules such as the ATG series of proteins and beclin-1, with mTOR serving as a distal autophagy gatekeeper (mTOR on: autophagy inhibition; mTOR off: autophagy promotion) [9].

\section{"Indeed, as shown in the electron microscopy images, human $T$ cells manufactured ex vivo in rapamycin were replete with autophagosomes..."}

Given the importance of autophagy in determining T-cell survival (reviewed in [10]), we evaluated whether rapamycin exposure during ex vivo T-cell culture induced autophagy and modulated T-cell survival. For these studies, we evaluated human $\mathrm{CD}^{+}{ }^{+} \mathrm{T}$ cells that were polarized to a Th1 cytokine phenotype. Indeed, as shown in the electron microscopy images, human $\mathrm{T}$ cells manufactured ex vivo in rapamycin were replete with autophagosomes; consistent with an

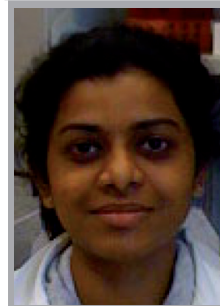

Shoba Amarnath

Author for correspondence: Experimental Transplantation \& Immunology Branch, National Cancer Institute, National Institutes of Health, Clinical Research Center, 3-East Labs, 3-3224, Bethesda, MD 20892, USA

Tel.: +13015949687

Fax: +13014803436 samarnath@mail.nih.gov

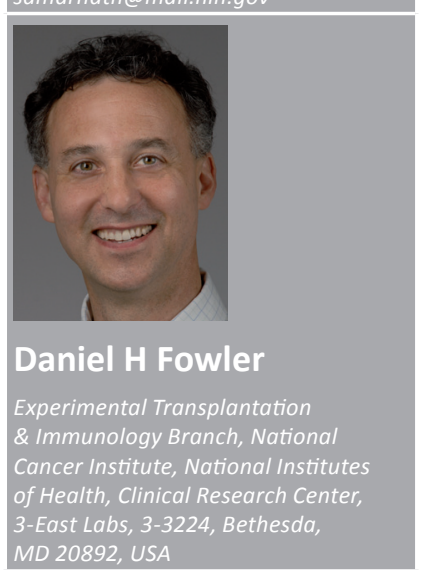

\section{Future Medicine parto}


autophagy mechanism, T cells knocked-down for Beclin-1 had reduced autophagosome formation during mTOR blockade (Figure 1). Initially, rapamycin was characterized as an agent that induces T-cell anergy even in the presence of costimulation [11]; in this light, it was somewhat paradoxical that the postautophagy, rapamycinresistant $T$ cells that we evaluated had increased in vivo function upon adoptive transfer into a human-into-mouse model of xenogeneic graftversus-host disease (GVHD) [12]. In these experiments, we also found that rapamycin-resistant $\mathrm{T}$ cells underwent mitochondrial autophagy (mitophagy), thereby resulting in reduced T-cell mitochondrial mass but increased mitochondrial function (more stable mitochondrial membrane potential). These results extended previous findings in human cell lines, where it was observed that autophagy altered mitochondrial biology in $\mathrm{T}$ cells, including a reduction in mitochondrial mass [13].

\section{"...rapamycin-resistant T cells underwent mitochondrial autophagy (mitophagy), thereby resulting in reduced T-cell mitochondrial mass but increased mitochondrial function..."}

In these experiments, we also observed that ex vivo manufacturing in rapamycin decreased T-cell free oxygen radical formation, was associated with reduced T-cell expression of proapoptotic $b c l-2$ family gene members $(B A X$ and $B A K)$, and yielded an antiapoptotic phenotype in rapamycin-resistant T cells [12]. We found that the antiapoptotic phenotype of rapamycin-resistant
T cells was relatively optimized: that is, such $T$ cells had similar engraftment and persistence characteristics as transgenic $T$ cells overexpressing antiapoptotic bcl-2. Importantly, we observed a nearly all-or-nothing phenomenon with respect to human T-cell engraftment and persistence in the xenogeneic GVHD model: that is, rapamycin-resistant $\mathrm{T}$ cells (but not control, costimulated $T$ cells) engrafted and persisted for months after adoptive transfer. In addition, with such long-term engrafting, rapamycin-resistant human $\mathrm{T}$ cells vigorously engrafted upon transfer to secondary immune-deficient murine hosts [Amarnath S, Unpublished Observation]; this latter observation is consistent with a conclusion that ex vivo rapamycin promoted long-term memory T-cell characteristics. These results run parallel to findings in models of malignant glioma [14] and tuberous sclerosis complex [15], where autophagy promotes tumor cell clonogenicity.

We have found that postautophagy, rapamycin-resistant $\mathrm{T}$ cells can be manufactured in both Th1- and Th2-polarizing conditions [16], thereby offering diverse opportunities for clinical translation. In this study, we found that ex vivo rapamycin promoted a $\mathrm{T}$ central memory phenotype; this result is consistent with the known linkage of mTOR and T-cell homing through modulation of KLF2 transcription factor levels [17] and with findings of the superior in vivo persistence of T central memory cells [18]. An ability to polarize towards both Th1 and Th2 phenotypes in the presence of high-dose rapamycin contrasts somewhat with results obtained using $\mathrm{T}$ cells genetically deficient in TORC1/ TORC2, where mTOR status was shown to

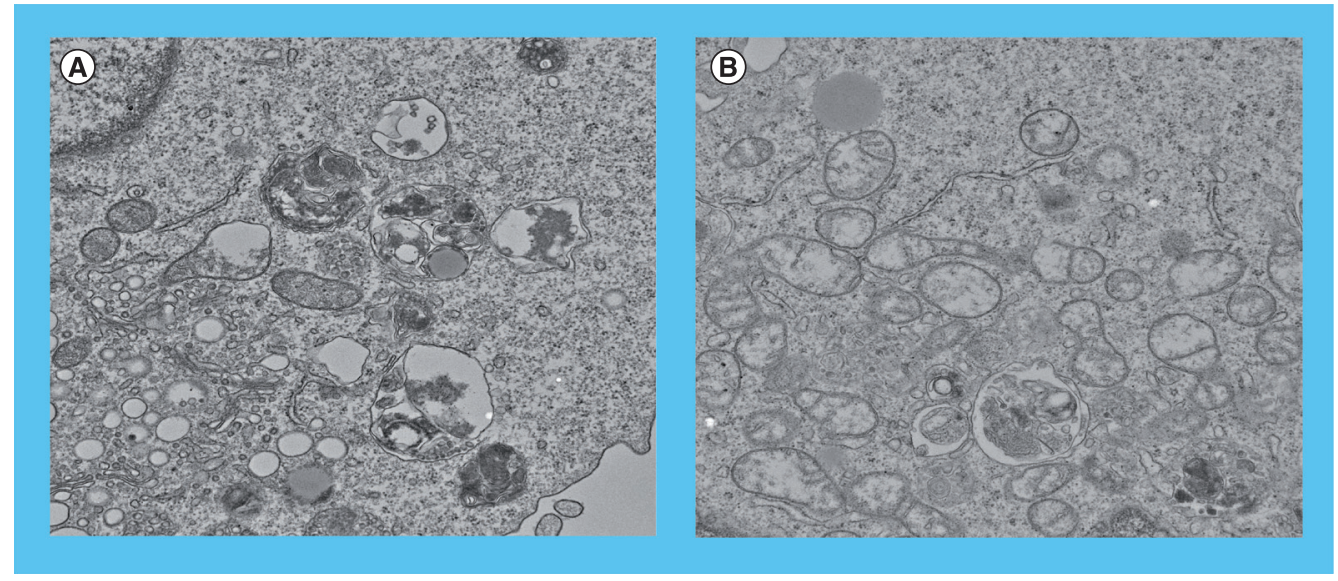

Figure 1. Rapamycin induces autophagy in human Th1 cells. CD4 ${ }^{+} \mathrm{T}$ cells were costimulated with anti-CD3, anti-CD28 and cultured for 6 days in media containing rhIL2 (20 IU/ml), IFN- $\alpha 2 b$ (1 million IU/ml) and rapamycin. (A) Resultant CD4+ Th1 cells expressed autophagosomes by electron microscopy. (B) Culture initiation with CD4 ${ }^{+}$T cells knocked-down for Beclin-1 expression by siRNA methodology had greatly reduced autophagy. 
influence T-cell polarization [19]; and, our findings differ somewhat from studies using T-cell culture without polarizing cytokine addition, where rapamycin was shown to promote Treg differentiation [20]. Because of the association of Th1-type cells with GVHD [21], rapamycinresistant $T$ cells may have application primarily in the autologous transplantation setting; along these lines, we have sponsored an Investigational New Drug Application with the US FDA for evaluation of rapamycin-resistant Th1 cells for immune reconstitution after autologous HCT therapy of multiple myeloma [101].

\section{“...rapamycin-resistant $T$ cells (but not control, costimulated T cells) engrafted and persisted for months after adoptive transfer."}

On the other hand, rapamycin-resistant Th2type cells may have clinical translational application in the setting of allogeneic transplantation. We found that allogeneic murine Th2 cells manufactured ex vivo in rapamycin prevented GVHD in an IL-4 dependent manner, and when combined with $\mathrm{T}$ cells that induced a Th1-type response, could yield a favorable balance between GVHD protection and mediation of graft-versustumor effects [22]. Furthermore, we found that antiapoptotic, rapamycin-resistant Th2-type cells prevented murine hematopoietic cell graft rejection [23]; in other studies, we demonstrated that host-based therapy with rapamycin-generated Th2 cells prevented graft rejection in a rat model of cardiac transplantation [24]. Based on these results, we have initiated a pilot clinical trial of rapamycin-resistant human Th2 cells in the setting of low-intensity allogeneic HCT for therapy of refractory hematologic malignancy [102]. Initial results, presented in abstract form $[25,26]$, indicate that this approach can promote alloengraftment and preserve antitumor effects with a low rate of GVHD.

\section{References}

1 Chakraverty R, Mackinnon S. Allogeneic transplantation for lymphoma. J. Clin. Oncol. 29(14), 1855-1863 (2011).

2 Robbins PF, Morgan RA, Feldman SA et al. Tumor regression in patients with metastatic synovial cell sarcoma and melanoma using genetically engineered lymphocytes reactive with NY-ESO-1. J. Clin. Oncol. 29(7), 917-924 (2011).

3 Porter DL, Levine BL, Kalos M, Bagg A, June $\mathrm{CH}$. Chimeric antigen receptor-modified

\section{“...rapamycin-resistant T cells can be} manufactured in both Th1- and Th2polarizing conditions ... thereby offering diverse opportunities for clinical translation."

In conclusion, through use of ex vivo methods of T-cell expansion that incorporate rapamycin and polarizing cytokines, autophagy can be harnessed for the generation of Th1- or Th2-type T cells that mediate increased in vivo effects upon adoptive transfer. This flexibility in generation of cross-regulatory Th1/Th2 subsets indicates that this approach may have application for both autologous and allogeneic transplantation. The beneficial effects of autophagy on T-cell function are certainly complex, and are probably mediated at least in part through alterations in mitochondrial biology, apoptotic tendency and effector maturation. Further studies will be required to more fully characterize the role of autophagy for enhancement of T-cell function and to evaluate whether alternative proautophagy agents may work in a different or synergistic manner with rapamycin.

\section{Acknowledgements}

The authors would like to thank FA Flomerfelt, Experimental Transplantation Immunology Branch, National Cancer Institute, National Institute of Health for his expertise in electron microscopy.

\section{Financial \& competing interests disclosure}

The authors have no relevant affliations or financial involvement with any organization or entity with a financial interest in or financial conflict with the subject matter or materials discussed in the manuscript. This includes employment, consultancies, honoraria, stock ownership or options, expert testimony, grants or patents received or pending, or royalties.

No writing assistance was utilized in the production of this manuscript.

T cells in chronic lymphoid leukemia. N. Engl. J. Med. 365(8), 725-733 (2011).

4 Levine BL, Bernstein WB, Aronson NE et al. Adoptive transfer of costimulated $\mathrm{CD}^{+}$ $T$ cells induces expansion of peripheral $T$ cells and decreased CCR 5 expression in HIV infection. Nat. Med. 8(1), 47-53 (2002).

5 Heslop HE, Slobod KS, Pule, MA et al. Long term outcome of EBV specific T cell infusions to prevent or treat EBV-related lymphoproliferative disease in transplant recipients. Blood 115(5), 925-935 (2010).
6 O'Shea JJ, Paul WE. Mechanisms underlying lineage commitment and plasticity of helper CD4 ${ }^{+}$T cells. Science 327(5969), 1098-1102 (2010).

7 Hinrichs CS, Borman ZA, Gattinoni L et al. Human effector $\mathrm{CD} 8^{+} \mathrm{T}$ cells derived from naive rather than memory subsets possess superior traits for adoptive immunotherapy. Blood 117(3), 808-814 (2011).

8 Kundu M, Thompson CB. Autophagy: basic principles and relevance to disease. Annu. Rev. Pathol. 3, 427-455 (2008). 
9 Walsh CM, Edinger AL. The complex interplay between autophagy, apoptosis, and necrotic signals promotes T-cell homeostasis. Immunol. Rev. 236, 95-109 (2010).

10 Lunemann JD, Munz C. Autophagy in $\mathrm{CD}^{+}$ T-cell immunity and tolerance. Cell Death Differ. 16(1), 79-86 (2009).

11 Powell JD, Lerner CG, Schwartz RH. Inhibition of cell cycle progression by rapamycin induces $\mathrm{T}$-cell clonal anergy even in the presence of costimulation. J. Immunol. 162(5), 2775-2784 (1999).

12 Amarnath S, Flomerfelt FA, Costanzo CM et al. Rapamycin generates antiapoptotic human Th1/Tc1 cells via autophagy for induction of xenogeneic GVHD. Autophagy 6(4), 523-541 (2010).

13 Colell A, Ricci JE, Tait S et al. GAPDH and autophagy preserve survival after apoptotic cytochrome $\mathrm{c}$ release in the absence of caspase activation. Cell 129(5), 983-997 (2007).

14 Fan QW, Cheng C, Hackett C et al. Akt and autophagy cooperate to promote survival of drug-resistant glioma. Sci. Signal. 3(147), RA81 (2010).

15 Parkhitko A, Myachina F, Morrison TA et al. Tumorigenesis in tuberous sclerosis complex is autophagy and p62/sequestosome 1 (SQSTM1)-dependent. Proc. Natl Acad. Sci. USA 108(30), 12455-12460 (2011).

16 Jung U, Foley JE, Erdmann AA et al. Ex vivo rapamycin generates $T h 1 / \mathrm{Tc} 1$ or $\mathrm{Th} 2 / \mathrm{Tc} 2$ Effector $\mathrm{T}$ cells with enhanced in vivo function and differential sensitivity to post-transplant rapamycin therapy. Biol. Blood Marrow Transplant. 12(9), 905-918 (2006).

17 Finlay D, Cantrell D. Phosphoinositide 3-kinase and the mammalian target of rapamycin pathways control T-cell migration. Ann. NY Acad. Sci. 1183, 149-157 (2010).

18 Berger C, Jensen MC, Lansdorp PM, Gough $\mathrm{M}$, Elliott C, Riddell SR. Adoptive transfer of effector $\mathrm{CD} 8^{+} \mathrm{T}$ cells derived from central memory cells establishes persistent $\mathrm{T}$-cell memory in primates. J. Clin. Invest. 118(1), 294-305 (2008).

19 Powell JD, Delgoffe GM. The mammalian target of rapamycin: linking T-cell differentiation, function, and metabolism. Immunity 33(3), 301-311 (2010).

20 Battaglia M, Stabilini A, Roncarolo MG. Rapamycin selectively expands $\mathrm{CD} 4{ }^{+} \mathrm{CD} 25^{+} \mathrm{FoxP}^{+}{ }^{+}$regulatory $\mathrm{T}$ cells. Blood 105(12), 4743-4748 (2005).

21 Fowler DH, Breglio J, Nagel G, Hirose C, Gress RE. Allospecific CD4 ${ }^{+}$, Th1/Th2 and $\mathrm{CD} 8+, \mathrm{Tc}^{2} / \mathrm{Tc} 2$ populations in murine GVL: type 1 cells generate GVL and type 2 cells abrogate GVL. Biol. Blood Marrow Transplant. 2(3), 118-125 (1996).

22 Foley JE, Jung U, Miera A et al. Ex vivo rapamycin generates donor Th 2 cells that potently inhibit graft-versus-host disease and graft-versus-tumor effects via an IL-4dependent mechanism. J. Immunol. 175(9), 5732-5743 (2005).
23 Mariotti J, Foley J, Jung U et al. Ex vivo rapamycin generates apoptosis-resistant donor Th2 cells that persist in vivo and prevent hemopoietic stem cell graft rejection. J. Immunol. 180(1), 89-105 (2008).

24 Amarnath S, Chen H, Foley JE et al. Host-based Th2 cell therapy for prolongation of cardiac allograft viability. PLoS ONE 6(4), E18885 (2011).

25 Mossoba ME, Mariotti J, Yan XY et al. T rapa cell clinical products contain a balance of minimally differentiated Th2/Th1 effector cells depleted of Treg cells. Blood 116(21), 158-159 (2010).

26 Fowler DH, Mossoba ME, Schuver BB et al. Adoptive transfer of Treg depleted donor Th1 and Th2 cells safely accelerates alloengraftment after low intensity chemotherapy. Blood 116(21), 230-231 (2010).

\section{Websites}

101 Th1/Tc1 immunotherapy following stem cell transplantation in multiple myeloma. http://clinicaltrials.gov/ct2/show/ NCT01239368

(Accessed 3 November 2011)

102 Donor stem cell transplant with no or low-intensity chemotherapy using sirolimus and treated immune cells to treat blood and lymph cancers.

http://clinicaltrials.gov/ct2/show/

NCT00074490

(Accessed 3 November 2011) 